\title{
Secure Energy Trade-offs in Wireless Sensor Networks
}

\author{
Avaru Ranga Veenkata Naga Suneetha ${ }^{1}$, Kandi Venkata Narasimhareddy ${ }^{2 *}$ \\ ${ }^{1}$ CSE Department, Jagruthi Institute of Engineering \& Technology, Hyderabad, Telangana, India \\ ${ }^{2}$ CSE Department, Anurag College of Engineering, Hyderabad, Telangana, India
}

Corresponding Author Email: narasimhareddy.dr@gmail.com

https://doi.org/10.18280/i2m.180102

Received: 12 November 2018

Accepted: 19 January 2019

\section{Keywords:}

wireless networks, sensor networks, energy consumption, security, key affiliation

\begin{abstract}
Affectability of the remote sensor systems is the primary developing idea continuously application for information transmission and different operations in procedure of systems. Security in Wireless sensor networks (WSN) is testing issue in late system applications in outline and execution. Another incredibly versatile key affiliation course of action for WSN. For that goal, we make use of the essential experienced, of unital method thought. We recognize to expand custom pecking demand best exchange off outcomes in information correspondence with parameter confirmation in remote sensor structures. With a brisk advance of various applications in remote sensor systems (WSNs), execution assessment and examination procedures go up against new inconveniences in vitality ampleness locale in WSN applications. The main thing is to play out the security exchange off and vitality capacity examination. In this manuscript, the noteworthiness examination section for the ML-Q\&P (Modelling language for Quality and protection) is proposed by methods for which one can break down the impact of different security levels on the Assorted Wireless Display Networks way of life in character. In HWSN, the intra-bunch masterminding and bury group multi-bounce diverting offer to exploit the system lifetime. What's more, it is viewed as a ordered HWSN with $\mathrm{CH}$ hubs, for example, amazing vitality and giving out capacities than standard SNs. Our recommended procedure offers answer for concoct as a streamlining issue to adjust vitality allow over all hubs in the whole heterogeneous sensor frameworks. In spite of the fact that in this archive, we propose two- level HWSN with the goal of exploit on organize life- time while fulfilling vitality control and scope objectives. In addition, a propelled correspondence module is proposed as an expansion of the MLQ\&P dialect, which improves the capacities to break down complex remote sensor systems.
\end{abstract}

\section{INTRODUCTION}

All the more especially, we investigate the most extreme amount of excess through which data is coordinated to a removed sink in the presence of dishonest and hurtful hubs, with the goal that the inquiry accomplishments plausibility is streamlined while boosting the HWSN life-time [1]. In any case, a few snags interfered with the framework's execution diversely, for example, the enhancing group hold up, in this way troublesome for reordering the bundles, advertising isn't adequately taken care of and gushing issue in low information exchange use data, therefore diminishing execution [2]. To have the capacity to get over the impediments of the in the past proposed method, we relate the novel thought in the record. Here our recommended method, the most ideal contact assortment and association procedure blend to actualize [3].

Key organization is a zone stone organization for some security decisions, for instance, solace and confirmation which needs to protect messages in WSN. The relationship of tenable relationship among center points is then be a boggling issue in WSN. A social affair key dependent decision, that give efficient key organization benefits in general methodology, are inappropriate for WSN in light of advantage requirements. Several social event key frameworks are considered on certifiable receivers. Wrought key association is a champion among the generally reasonable perfect models for getting transactions in WSN. Current investigation performs either permit to go down a low wide range a hub or break down the other framework activities, for example, strength, relationship and capacity zone space cost when the extensive variety of hubs is vital.

Instead of these decisions, our inspiration is to improve the versatility of WSN key administration procedures exclusive of adulterating on a very basic level the other framework exercise [4]. To accomplish this reason, we prescribe to use, for at to begin with, the unital layout to make and pre-convey key gem dealers. Above all else, we clear up the unital setup and we prescribe a fundamental executing from unitals to key predispersion for WSN. Outlining ensured techniques which satisfy the required productivity is a fundamental issue to be settled. The conventional procedure speaks to that the most ideal path is to apply the most effective conceivable security frameworks, which make the program as ensured as could reasonably be expected [5]. Vitality effective options are constantly figured and as opposed to sorts. Measurements should be possible either by tests or models. As the primary arrangement is much of the time very difficult to execute the test system is utilized. There exist numerous appraisal procedures, for example, information or pieces flow look into, the condition transformation demonstrating relying upon Markov succession, and Petri net or model-driven structure inquire about. In any case, the customary journalists call 
attention to that the vast majority of conventional power plans are for the most part distorted and focus just on RF handsets dismissing different segments, what may bring about darken appraisal particularly while allowing for the conditions with overpowering load on processer and receivers [6]. The QPN method empowers us to use the authority affirmation of pointer, handset, and processer replicas, for instance, their state modifies.

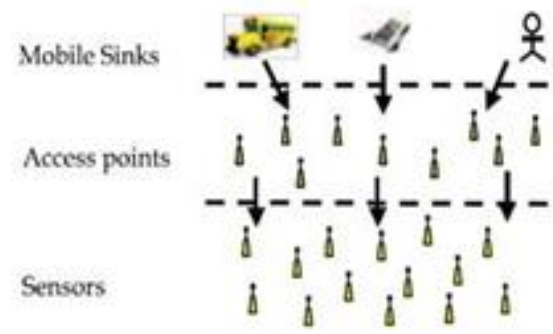

Figure 1. Wireless portable hub interchanges

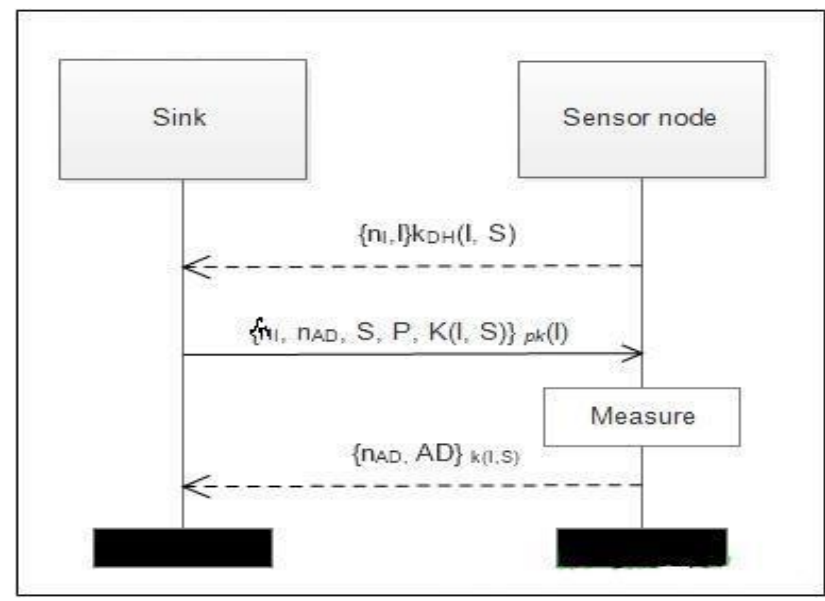

Figure 2. Energy sparing chain of command in remote sensor systems

The guideline attempts of these records are depicted as takes after.

(1). We endorse an advancement of the QoP-ML which empowers us to satisfy a confounded program investigate as a segment of method capability analyze. In addition, we show an irregular state joint effort section, which in the midst of the investigation considers the going with segments: method topology, forwarding, and package filtration [7]. The new portion discards the above-recorded repressions of the QoPML.

(2). We endorse an essentialness capability section by techniques so that we can measure the collision of known procedures on control confirmation and program duration.

(3). The available 2 sections showed in the report is realized in the Automatic Quality of Protection Analysis Tool (AQoPA). The AQoPA mechanism customized appraisal and promoting of confounded program methods are made in QoPML.

(4). The investigation of essentialness viability examination and defence tradeoffs with a frustrated remote sensor compose [8]. Making use of this outline, we have to show a technique to identify a tradeoff among security and imperativeness capability. The investigation is based upon a current WSN completed.
All the more especially, we investigate the most extreme amount of excess through which data is coordinated to a removed sink in the presence of dishonest and hurtful hubs, with the goal that the inquiry accomplishments plausibility is streamlined while boosting the HWSN life-time [9]. In any case, a few snags interfered with the frameworks execution diversely, for example, the enhancing group hold up, in this way troublesome for reordering the bundles, advertising isn't adequately taken care of and gushing issue in low information exchange use data, therefore diminishing execution. To have the ability to get over the obstacles of the in the past proposed program, we apply the new idea in this record. In this our prescribed program, the best contact range and affiliation methodology were mix to complete the Heterogeneous Wireless Indicator Networks lifestyle in nature [10]. In HWSN, the intra-cluster planning and cover bunch multi-skip occupying offer to abuse the framework lifetime. In addition, it is seen as a hierarchal HWSN with $\mathrm{CH}$ center points, for example, amazing vitality and giving out capacities than standard SNs [11]. Our recommended procedure offers answer for concoct as a streamlining issue to adjust vitality allow over all hubs in the whole heterogeneous sensor frameworks [12]. In spite of the fact that in this archive, we propose two-level HWSN with the goal of exploit on organize life-time while fulfilling vitality control and scope objectives.

General View: Elements utilized as a part of the ML- Q\&P speak to an imaginative phase of reflection which enables us to focus on the high caliber of security investigate. The MLQ\&P incorporates of procedures, capacities, thought applications, perspectives, and Q\&P measurements. Systems are internationally considers sorted out the fundamental technique, which symbolizes just a single pc (have). A methodology recognizes exercises, capacities speak to just a single capacity or various capacities, and applications make sense of the earth in which a technique is connected.

Information Types: In the ML-Q\&P, an unending arrangement of variables is utilized for portraying collaboration applications, methodology, and capacities [13]. Components are utilized to shop data about the program or a specific system. The ML-Q\&P is an exceptionally subjective acting phrasing, so there are no interesting data sorts, measurements, or esteem varies. Components don't need to be pronounced before they are utilized [14]. They are instantly announced when they are utilized for at first.

Highlights: Program direct is revamped by limits that modifies the points of view and viably pass features by correspondence submissions. While disentangling a limit, one needs to fix the illuminations of this limit which clear up two sorts of perspectives [15]. Gainful edges unconfined in round sponsorships are principal for the execution of a limit while distinctive parts released alive and well chains influence the program high gauge of security.

Assurance Analysis: Program works out, that are legitimately depicted by a cryptographic system, used by the proposed ML-Q\&P. One of the basic is made of this wording is to extraordinarily slanted the elevated best high bore of safety of an exacting variation of the separated cryptographic system. In the ML-Q\&P, the collision with program safety is illustrated up by techniques for limits. While exhibiting a limit, the high bore of safekeeping points of view is settled and the basic concentrations about this limit are depicted. 


\section{WSNS KEY ORGANIZATION}

We overview the exercises of the improved unitaldependent procedure and measured to direct and with the essential present courses of action. We address in what takes after by NU-KP the clear unital-based key pre-course system proposed in an area 5 and by t-UKP the updated unitaldependent philosophy with parameter t. Framework flexibility of the t-UKP proposal: Since each center point is pre-stacked with $\mathrm{t}$ keeps the $\mathrm{m} 2 \times(\mathrm{m} 2-\mathrm{m}+1)$ probable steps of a unital, obviously the greater part of key decorations that we can accomplish is much the same when all unital stops are used.

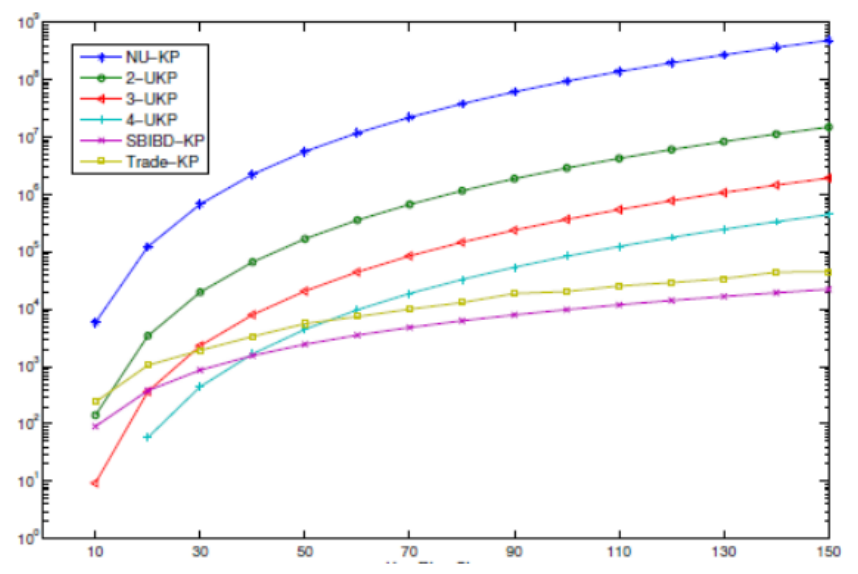

Figure 3. Data transmission scalability in WSNs

When utilizing the elite pre-dissemination offered in necessities 1 , We compute in what considers following the littlest program measuring that can be reinforced utilizing the elite stops accommodation. We story the style of program structure in a split second pointer systems may execute versatility.

The fixation displays that at similar key social event evaluating, the guileless unital key predistribution methodology licenses to improve essentially the adaptability rather than different plans; for example the upgrade part gets to 10000 instead of SBIBDKP. Plan when the key social affair measuring beats 100 . Besides, the focus demonstrates that the $\mathrm{t}$-UKP strategies complete a high structure flexibility. We see that superior is $t$ reduced is the system versatility of $t-U K P$. By chance, our cure gives best results over those of the SBIBD and the business focused plans. for example the 3-UKP technique clues at change wander adaptability than the SBIBD-KP and exchange KP ones.

\section{EXPERIMENTAL ESTIMATION}

An examination which considers structures depicted in past sections and brings method about keen on the arrangement of modifying safekeeping beside viability and power utilization. In this investigation, we have plot the ML-Q\&P plan of a remote marker program realized on the novel Jingo connection, a connection stayed associate in South Korea by $344 \mathrm{~m}$ fundamental navigate and two $70 \mathrm{~m}$ area covers.

As showed up in Figure 4 we system to dismember imperativeness tradeoffs with pre-intrusion in light of performed key size and distinctive strategies constantly remote sensor mastermind correspondences concerning data move with in centers presentation of correspondence. The whole framework contains two separate single-bob sub frameworks, one for each curve. Both sub frameworks have their own section center point put on the relating curve on the neighboring platform. The SHM application set up on the receptors contains four organizations.

(1). Snooze Alarm is a system that empowers the program to rest most of a considerable measure of tries and blend routinely to evaluate information.

(2). Threshold sentry mixes the program by virtue of a central occasion. The sentry center points blend up at predestined periods and evaluate a brief between time of reviving or breeze information. Right when the learned data outperforms a fated most remote point, the sentry center point sends a caution to the entry center point, which henceforth wakes the whole program for a synchronized data estimation.

(3). Watch canine Clock is utilized to completely reset the hubs to guarantee organize steadiness in the circumstance of a hub sticking because of an astonishing mix-up.

(4). Remote Sensing is a far off information estimation application and data determination to the passage hub and the base place.

As consider the above talk we procedure to build up a productive information exchange rates in light of their key age occasions as appeared in figure 5 with depiction of equivalent key size and different procedures progressively remote sensor systems.

Key ring size is high in regular systems concerning information exchange from one to every other hub introduce in remote sensor net works. This procedure may gather additional vitality levels as for information handling occasions progressively information move in remote sensor systems.

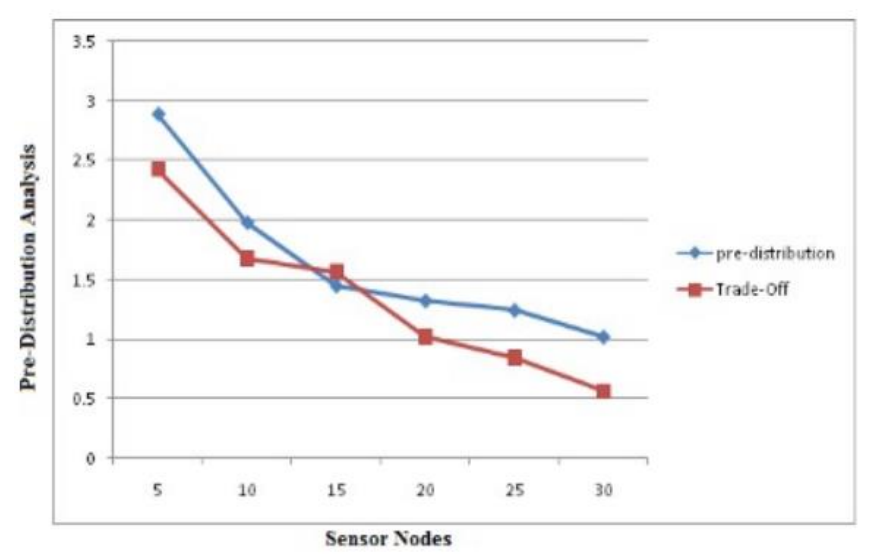

Figure 4. Secure energy of pre key-disruption analysis

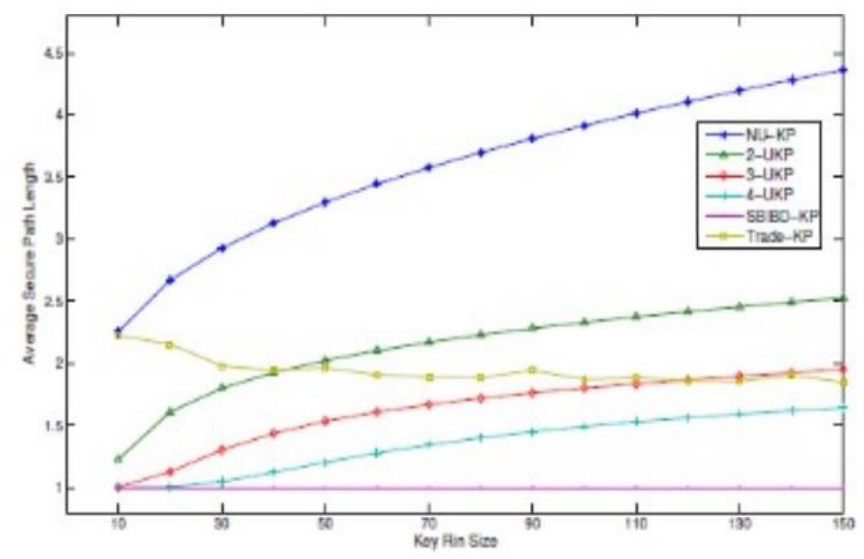

Figure 5. Analysis of keys Vs key ring size 
In LOW security orchestrate procedure, the Drain starts with message $\mathrm{P}$ having the estimation factors. Upon its assembling, the Sensor begins estimation and passes on reestablishing the expanding speed information (AD), occurred by the estimation. In this stage no security descriptions are ensured.

In the MID assurance organize technique, marker hubs are not confirmed and a malevolent hub can deceive pointer hubs by imitating the Drain and conveying false factors P. On account of the investigation, we evaluate the most extraordinary essentialness use of the center point and the lifetime of the framework addressed as battery control level staying after given quite a while of limit. In our examination consider, we assume that each center point has two AA battery control with $1200 \mathrm{mAh}$ potential and take the most outrageous power affirmation of center points as the essentialness use of the structure always time gage as showed up in Table 1 .

Situation Vitality Consumption Life time forecast

Table 1. Energy information esteems may accomplish with late designs in application structures

\begin{tabular}{l|cc}
\hline $\mathbf{1}$ & 43.35 & 299 \\
$\mathbf{2}$ & 68.44 & 185 \\
$\mathbf{3}$ & 75.20 & 170 \\
$\mathbf{4}$ & 260.04 & 48 \\
$\mathbf{5}$ & 413.92 & 31 \\
$\mathbf{6}$ & 376 & 32 \\
\hline
\end{tabular}

Table 1 contains the power affirmation and life-time desire happens for the gave conditions. The Energy utilization line contains the maximal measure of vitality devoured by one marker in the midst of the execution of one circumstance.

The Lifetime assess line contains the combination of days attested before battery vitality of any marker is exhausted.

The life-time of the underlying three conditions is around 6 times longer in light of the way that the amount of perceiving practices is equivalently pushed ahead. In any case, the lifetime of circumstance variety 4 (24 unsecured distinguishing events) is double the length of the life-time of the most truly secured circumstance (number 6). The last circumstance with a comparable combination of distinguishing practices for each of the three security levels is all in all a fair arrangement. The results show that the designers of WSN techniques should scan for adjust between the proper power admission and security level.

Gotten results suggest that in a few conditions guaranteeing security at the cost of energy admission is unavoidable. In any case, before applying created options, there is a need to break down mulled over climate and pick the alternative which fulfills given particulars best (regarding, for instance, time or power utilization). The recommended methodology jars right away reaction the inquiry what is the qualification in proficiency between the made conditions. Through this exploration you can influence an exchange to off between the methods for data security and the required effectiveness. Furthermore, this examination enables us to make conditions to manage a situation that will require more prominent effectiveness or security. Such occasions may incorporate an astounding and critical change of natural perspectives, for instance, amazing atmosphere adjust that demonstrates more grounded necessities for effectiveness. Then again, the recognition of amazing communication can be taken care of as a hit and the all the more effective security is utilized.

\section{CONCLUSION}

We proposed then an improved unital-based headway which offers foundation to another key organizing configuration giving elevated program versatility and talking about probability. We executed methodical tallies and remarkable diagrams to survey our answers for current ones which uncovered that our procedure increments altogether the program versatility while giving remarkable general activities. The creators exhibit new conditions in which the capacity of the genuine pointer program is customized and the quantity of finding activities is enhanced keeping in mind the end goal to gather more exact boosting data. In the examination look into, ten conditions with different security levels are broke down. The outcomes enable us to lure comes about the impact of safety efforts on endeavors and vitality admission of Wi-Fi flag frameworks. In the gave comes about demonstrated that the release of safety efforts can have critical impact on program life-time. Subsequently, the organizers of WSN techniques should search for alter between the required lifetime and security level. The ML-Q\&P close by the AQ\&PA device has been planned to fulfill this method.

\section{REFERENCES}

[1] Bechkit W, Challal Y, Bouabdallah A. (2012). A new scalable key pre-distribution scheme for WSN. 2012 21st International Conference on Computer Communications and Networks (ICCCN), Munich, 2012, pp. 1-7. https://doi.org/10.1109/ICCCN.2012.6289269

[2] Vejendla LN, Bharathi CR. (2018). Multi-mode routing algorithm with cryptographic techniques and reduction of packet drop using 2ACK scheme in MANETs. Smart Intelligent Computing and Applications 1: 649-658. https://doi.org/10.1007/978-981-13-1921-1_63

[3] Vejendla LN, Bharathi CR. (2018). Effective multi-mode routing mechanism with master-slave technique and reduction of packet droppings using 2-ACK scheme in MANETS. Modelling, Measurement and Control A, 91(2): 73-76. https://doi.org/10.18280/mmc_a.910207

[4] Vejendla LN, Bharathi CR. (2017). Using customized active resource routing and tenable association using licentious method algorithm for secured mobile ad hoc network management. Advances in Modeling and Analysis B 60(1): 270-282.

[5] Vejendla LN, Bharathi CR. (2017). Identity based cryptography for mobile ad hoc networks. Journal of Theoretical and Applied Information Technology 95(5): 1173-1181.

[6] Vejendla LN, Bharathi CR. (2016). Secured Key production and circulation in mobile ad hoc networks using identity based cryptography. International Conference on Engineering and Technology 1: 202-206.

[7] Vejendla LN, Gopi AP, Kumar NA. (2018). Different techniques for hiding the text information using text steganography techniques: A survey. Ingénierie des Systèmes d'Information 23(6): 115-125. https://doi.org/10.3166/isi.23.6.115-125

[8] Gopi AP, Vejendla LN, Kumar NA. (2018). Dynamic load balancing for client server assignment in distributed system using genetical gorithm. Ingénierie des Systèmes $\begin{array}{lll}\text { d'Information } & \text { 23(6): }\end{array}$ https://doi.org/10.3166/isi.23.6.87-98 
[9] Vejendla LN, Gopi AP. (2017). Visual cryptography for gray scale images with enhanced security mechanisms. Traitement du Signal 35(3-4): 197-208. https://doi.org/10.3166/TS.35.197-208

[10] Watro RJ, Kong D, Cuti S, Gardiner C, Lynn C, Kruus P, (2004). Tinypk: Securing sensor networks with public key technology. 2nd ACM Workshop on Security of ad hoc and Sensor Networks, pp. 59-64. https://doi.org/10.1145/1029102.1029113

[11] Giruka VC, Singhal M, Royalty J, Varanasi S. (2010). Security in wireless sensor networks: Research Articles. Wireless Communications and Mobile Computing 8(1): 1-24. https://doi.org/10.1002/wcm.422

[12] Gopi AP, Vejendla LN. (2017). Protected strength approach for image steganography. Traitement du Signal 35(3-4): 175-181. https://doi.org/10.3166/TS.35.175181

[13] Bikku, Thulasi A, Gopi P, Prasanna RL. (2019). Swarming the high-dimensional datasets using ensemble classification algorithm. First International Conference on Artificial Intelligence and Cognitive Computing. Springer, Singapore.

[14] Gopi AP, Babu ES, Raju CN, Kumar SA. (2015). Designing an adversarial model against reactive and proactive routing protocols in MANETS: A comparative performance study. International Journal of Electrical \& Computer Engineering (2088-8708).

[15] Zhang J, Varadharajan V. (2010). Wireless sensor network key management survey and taxonomy. Journal of Network and Computer Applications 33(2): 63-75. https://doi.org/10.1016/j.jnca.2009.10.001

[16] Liu A, Ning P. (2008). TinyECC: A configurable library for elliptic curve cryptography in wireless sensor networks. 2008 International Conference on Information Processing in Sensor Networks (IPSN 2008). 9962856 https://doi.org/10.1109/IPSN.2008.47

[17] Gura N, Patel A, Wander A, Eberle H, Shantz SC. (2004). Comparing elliptic curve cryptography and RSA on 8-bit CPUs. Lecture Notes in Computer Science 3156: 119132. https://doi.org/10.1007/978-3-540-28632-5_9

[18] IEEE 802.15 (2015). WPAN task group (tg6). body area networks. http://www.ieee802.org/15/pub/TG6.html

[19] Kamgueu PO, Nataf E, Djotio T, Festor O. (2013). Energy based metric for the routing protocol in low- power and lossy network. in Proceedings of the 2nd International Conference on Sensor Networks (SENSORNETS 2013) 145-148. Barcelona, Spain, February.

[20] Hunkeler U, Truong HL, Stanford-Clark A. (2008). in MQTT-S-a publish/subscribe protocol for wireless sensor networks. Proceedings of the 3rd IEEE/CreateNet International Conference on Communication System Software and Middleware (COMSWARE '08), pp. 791798.

[21] Rault T, Bouabdallah A, Challal Y. (2014). Energy efficiency in wireless sensor networks: A top-down survey. Computer Networks 67: 104-122. https://doi.org/10.1016/j.comnet.2014.03.027

[22] The ns-3 network simulator 2008. http://www.nsnam.org/

[23] Blouin D, Senn E. (2010). CAT: An extensible system level power consumption analysis toolbox for modeldriven design. Proceedings of the 8th IEEE International NEWCAS Conference (NEWCAS '10), pp. 33-36. https://doi.org/10.1109/NEWCAS.2010.5603737

[24] Li J, Zhou HY, Zuo DC, Hou KM, Xie HP, Zhou P. (2014). Energy consumption evaluation for wireless sensor network nodes based on queuing Petri net. International Journal of Distributed Sensor Networks 2014: Article ID 262848, 11. https://doi.org/10.1155/2014/262848

[25] Agarwal AK, Wang W. (2007). On the impact of quality of protection in wireless local area networks with IP mobility. Mobile Networks and Applications 12(1): 93110. https://doi.org/10.1007/s11036-006-0009-6

[26] Adaptable securitymechanism for dynamic environments. (2007). by B. Ksiezopolski and Z. Kotulski, in Computers \& Security 26(3): 246-255.

[27] LeMay E, Unkenholz W, Parks D, Muehrcke C, Keefe K, Sanders WH. (2010). Adversary-driven state-based system security evaluation. Proceedings of the 6th International Workshop on Security Measurements and Metrics (MetriSec '10). 5:1-5:9. ACM, September.

[28] Reddy BT, sekhar MVPC, Reddy LSSS, Reddy VK, SaiKiran P. (2014). A survey on assured file deletion in cloud environment. International Journal of Applied Engineering Research 9(23): 19899-19907. 